\title{
La importancia del DOI y el formato HTML en la difusión del conocimiento médico y científico actual
}

\section{The importance of DOI and HTML format in the current diffusion of medical and scientific knowledge}

\section{Claudia RAmÍREZ-RENTERÍA*}

Unidad de Investigación en Enfermedades Endocrinas, Hospital de Especialidades, Centro Médico Nacional Siglo XXI, Instituto Mexicano del Seguro Social, Ciudad de México, México

«La ciencia no conoce países, porque el conocimiento pertenece a la humanidad y es la antorcha que ilumina al mundo»

Luis Pasteur

La adquisición, procesamiento y difusión del conocimiento médico ha cambiado en los últimos años. La medicina basada en la experiencia de un solo médico ha sido invalidada por la medicina basada en evidencia ${ }^{1}$, en el estudio sistemático y científico de los hechos, de tal forma que todo lo que se dice en cualquier foro académico y científico (incluso en una clase, un congreso o frente a un paciente) está sujeto a comprobación por parte del auditorio y las discusiones más ricas las gana el que tiene el artículo de mayor peso científico o la mayor calidad de evidencia. La ciencia se ha especializado hasta el punto que se requieren varios (incluso decenas de colaboradores) para publicar un artículo, lo cual abre el camino a la cooperación, la inclusión y la democratización de la ciencia a niveles que no se hubieran sospechado (o permitido) hace un par de décadas ${ }^{2}$. Las leyes han cambiado, la ciencia ha cambiado y es obligatorio para los instrumentos de divulgación científica, incluyendo las revistas médicas, adaptarse a estos cambios sin perder sus valores y principios.

La cantidad de revistas médicas y publicaciones por tema se han incrementado exponencialmente en las últimas décadas ${ }^{3}$ y es cada vez más fácil obtener textos de otros países, escritos en idiomas que anteriormente no figuraban en el acervo científico internacional por no ser de origen anglosajón o en idioma inglés. Debido a que persisten serios sesgos de publicación por parte de las principales editoriales científicas y a que los costos de publicación o compra de algunas revistas son increíblemente elevados (sin que esto se traduzca en mayores recursos para los autores), existen tendencias actuales a buscar revistas y publicaciones de acceso gratuito (open access), lo cual favorece la difusión de muchos artículos $^{4}$. Además, los autores e instituciones están considerando los beneficios de adquirir suscripciones que permitan la descarga gratuita de artículos para sus empleados y alumnos, lo que también ha ayudado a difundir textos que otrora se ignoraban sistemáticamente. Actualmente son pocas las
Correspondencia:

*Claudia Ramírez-Rentería

E-mail: clau.r2000@gmail.com
Fecha de recepción: 06-01-2020

Fecha de aceptación: 11-01-2020

DOI: 10.24875/RME.20000002
Disponible en internet: 25-02-2020 Rev Mex Endocrinol Metab Nutr. 2020;7:5-9

2462-4144 / @ 2020 Sociedad Mexicana de Nutricion y Endocrinologia, AC. Publicado por Permanyer. Este es un artículo open access bajo la licencia CC BY-NC-ND (http://creativecommons.org/licenses/by-nc-nd/4.0/). 
revistas que no solamente son gratuitas para autores y lectores, ya que obtienen fondos de sociedades, industrias farmacéuticas y gobiernos, sino que también garantizan la calidad de sus artículos al ser evaluados estos por pares de manera transparente y eficaz ${ }^{5}$.

Aun con todas sus limitantes, el acceso a internet permite que la mayoría de los estudiantes, médicos e investigadores puedan evaluar una gran cantidad de publicaciones de manera inmediata, las apliquen a sus labores cotidianas y las utilicen de referencia para sus propias investigaciones, gracias al uso de los dispositivos portátiles ${ }^{6}$. Labores que antes suponían meses de búsquedas en bibliotecas físicas, gastos y bajas tasas de éxito, ahora pueden realizarse en unos minutos desde cualquier parte del mundo que cuente con una red de internet. El registro de la propiedad intelectual, el determinar el momento exacto en el que se publicó cierta información y rastrear las veces que se ha dado el crédito correspondiente a estas publicaciones, generan la necesidad de tener publicaciones que garanticen todos estos derechos, además de la calidad científica. Las revistas médicas solían ser siempre un recurso fiable de información, sin embargo la gran cantidad de información disponible actualmente supone algunas desventajas:

- Se requiere formar un criterio para seleccionar las lecturas más adecuadas para un propósito específico, lo cual requiere habilidades de lectura, comprensión y manejo de estadística médica que aún no se enseñan a profundidad en todas las universidades.

- Se necesitan recursos económicos personales, grupales o institucionales para acceder a información que se encuentra en modalidad de «pago por uso» (pay per view).

- La habilidad de búsqueda está limitada a la capacidad de adaptación a la tecnología, ya que aunque se tenga conocimiento del tema, usar un «buscador» o "términos MeSH» adecuados también requiere entrenamiento ${ }^{7}$.

- Existen editoriales «depredadoras», que invitan a los autores y cobran por publicar artículos sin rigor científico, sin revisión por pares y con baja calidad, y que son a veces difíciles de distinguir de una revista que sí cumple estos requisitos ${ }^{8}$.

Por lo anterior, los investigadores buscan la seguridad de una revista «indizada» o arbitrada, definida como una publicación periódica de investigación que demuestra una alta calidad y ha sido listada en alguna base de datos de consulta mundial, por lo que finalmente puede tener un mayor índice de impacto. Para poder ser indizada, una revista debe cumplir varios requisitos e indicadores de calidad, que son cada vez más numerosos y estrictos.

La Revista de la Sociedad Mexicana de Nutrición y Endocrinología comenzó este proceso desde hace algunos años y lo concluyó en el 2018 gracias al uso de la plataforma en internet para el sometimiento de artículos. La plataforma permite: llevar un registro fidedigno de los artículos sometidos, tiempos de revisión, porcentaje de aceptación, seguimiento del arbitraje anónimo al que se someten los artículos por parte de varios integrantes del comité editorial antes de la toma de decisiones y la calidad del mismo comité. Es por esto que la revista se encuentra ya indizada en listas como Latindex e IMBIOMED y está en lista de revisión para PubMed.

Sin embargo, aun cuando una revista cumpla todos estos requisitos y sea de excelente calidad, la probabilidad de que sus artículos sean encontrados en una búsqueda de internet dependen, entre otras muchas cosas, de los algoritmos que utilizan los buscadores para encontrar la información ${ }^{9}$. La codificación de la plataforma, creada por los ingenieros, debe equilibrar la seguridad de la información contenida en la página con la facilidad para localizar y utilizar los textos contenidos en ella ${ }^{10}$. Por ejemplo, a más gráficos, diseño complejo, propaganda y seguridad tenga una página, más probable es que sea bloqueada por antivirus o que no se descargue adecuada o rápidamente en cualquier dispositivo, haciéndola estéticamente agradable, pero técnicamente difícil de usar. Varios estudios han demostrado que las páginas que tardan unos cuantos milisegundos de más en cargarse tienen una probabilidad menor de ser abiertas por los usuarios de internet ${ }^{11}$. Actualmente, la mayor parte de la información médica se obtiene de buscadores generales, no buscadores científicos específicos, 
debido a que su búsqueda es mucho más amplia y rápida ${ }^{12}$. Las palabras clave que escribimos en ese buscador son importantes para encontrar un texto, pero también el idioma en el que lo escribimos y el número de palabras, e incluso los errores de sintaxis y ortografía influyen en los resultados obtenidos. Un buscador básico utiliza las letras, más que las palabras, en un orden específico para hacer una búsqueda, los buscadores más avanzados generan "algoritmos», que son modelos o fórmulas que permiten al sistema interpretar con mayor libertad el orden de las letras y las palabras o la similitud de estas palabras en otros idiomas para obtener más y mejores resultados aun cuando el usuario cometa errores y los sugiere como «tal vez quisiste decir...» y nos da opciones para una «búsqueda avanzada» o más refinada ${ }^{12}$. Pero por muy flexible que el algoritmo sea, no podrá detectar ciertas páginas si no están en un formato específico que permita todas estas lecturas. Una manera de garantizar que la información científica sea localizada por los algoritmos sin requerir grandes cantidades de memoria o accesos especiales ha sido el crear y generalizar el uso de dos formatos: el DOI (digital object identificator) y el formato HTML (hypertext markup language $)^{13}$. El formato DOI es un «identificador persistente», se asigna a cualquier resultado científico comprobable, siempre y cuando tenga una dirección de internet; esto puede incluir pósteres, presentaciones en congresos y artículos médicos, a los cuales se les asigna un número único. Es una especie de certificado de nacimiento, que no cambia aun cuando las revistas cambien de sitio web, nombre o editorial, por ejemplo ${ }^{14}$. Los números en todo el mundo se almacenan en un solo sitio web de DOI para todos los archivos científicos, el cual no cambia con el tiempo. El número lo asigna la International DOI Foundation por medio de DataCite y otras 10 agencias. Las instituciones y revistas contratan a las agencias para generar sus propios números DOI y almacenarlos en esta página. La dirección del DOI tiene una estructura específica que se almacena en doi.org (llamado proxy) y tiene un prefijo que inicia con el número 10 y un punto, seguido de un registro específico para la institución, luego una diagonal que separa al sufijo, el cual es elegido por el registrante y se recomienda que no contenga ninguna «codificación

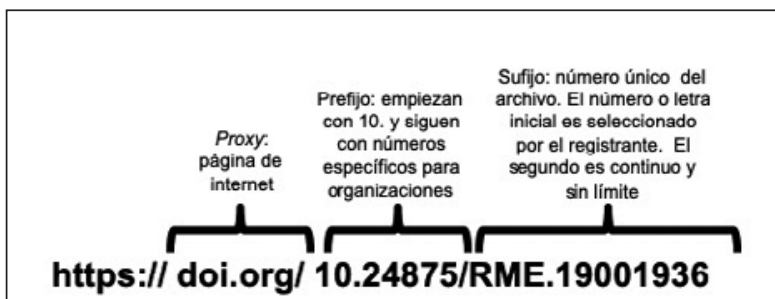

Figura 1. Estructura de una dirección con un número DOI. La estructura permite su localización dentro de la página de almacenamiento de DOI, la revista médica e incluso buscadores generales de internet sin que se pierda, aunque la página o la revista dejen de existir (adaptada de Rosenblatt, 199715).

semántica», es decir letras, símbolos, volumen o año de la revista, caracteres especiales del idioma o algún otro texto que sea complejo de traducir y utilizar en otros idiomas (incluso en lenguaje computacional) y que solamente dirijan hacia la página de la publicación en DOl; así si la página cambia o la revista se cierra, la conexión no se pierde porque no depende de la revista (Fig. 1) ${ }^{15}$.

El uso del DOI se ha extendido hasta el punto en el que las nuevas fórmulas para citar artículos, como las de la APA (American Psychological Association), incluyen la manera apropiada de citar un artículo con DOI. En la versión 2017 se sugiere: Apellido 1, Inicial. Apellido 2, Inicial., y Apellido 3, Inicial. (Año). Título del artículo. Nombre completo de la revista, volumen(número), página 1- última página. http:// dx.doi.org/10.xxxx/xxxx.xx

Por otra parte, el archivo simplificado HTML es una codificación que «marca» el texto para que se pueda ver con cierto tamaño de letra, color y fuente en una página específica de internet, es decir, que le da indicaciones de cómo proyectar el texto en cada pantalla y sea legible sin importar si es una pantalla gigante o de celular ${ }^{13}$. Ha resultado una herramienta útil para evaluar la transparencia de las búsquedas y publicaciones ${ }^{16}$, y también sirve para que los textos que «suben» las editoriales a su página no se «desconfiguren» aunque el editor lo haya mandado desde diferentes procesadores de texto o diferentes sistemas operativos. El HTML permite el almacenamiento de los archivos en menor espacio de memoria comparado con formatos como PDF, aunque nos da la posibilidad de descargar el artículo en este $u$ 
otro formato. Una ventaja científica importante del formato HTML es que permite que las páginas web muestren mucha más información de la que se puede ver en un PDF bidimensional ${ }^{17}$. Se pueden colocar vínculos a otras páginas de internet, material suplementario, vídeos, animaciones y propaganda sin que se pierda el formato para una lectura adecuada incluso en un dispositivo pequeño como un teléfono. Muchas revistas utilizan este formato para promocionar otros artículos similares, la producción científica de los autores, dar acceso a tablas o imágenes en dos ventanas al mismo tiempo, e incluso se pueden descargar documentos de procesadores de texto o paquetería estadística que puedan usar otros investigadores para corroborar los datos o colaborar con los autores. Todo esto de acuerdo con los lineamientos de la revista y los permisos otorgados por los autores. El formato HTML permite también el uso del texto (copiar y pegar) para usarlos en citas y referencias con mayor facilidad, conectan a los programas de referencia automática que facilita descargar las bibliografías y usarlas en cualquier formato $y$, a diferencia del DOI, también permite la «codificación semántica», es decir, permite a la computadora «darse cuenta» de que lo que está en esa página es texto que puede leer e interpretar y no solo una imagen; los algoritmos de los buscadores pueden entonces leer el texto, mostrarlo en los resultados y el usuario puede usar el texto sin importar el idioma en el que están escritos y sin que aparezcan caracteres especiales ilegibles. Estamos familiarizados con la codificación semántica en la vida cotidiana, es la que permite que al abrir una página en otro idioma, nuestra computadora abra una pequeña ventana en la que se nos ofrece «traducir» el texto de esa página al español de manera automática ${ }^{18}$. En términos sencillos, el HTML permite que un investigador en China, usando un navegador común, con una computadora e internet promedio, pueda encontrar un texto mexicano, escrito en español, copiarlo y pegarlo en un traductor y poder utilizar la información que de otra manera no habría logrado obtener hace unos años. Esto, sin duda, aumenta la visibilidad internacional de las revistas, por lo cual es un gran avance para nuestra revista que se cuente con estos dos formatos adicionales desde diciembre de 2019.
La Revista Mexicana de Endocrinología, Metabolismo y Nutrición, originalmente llamada Revista de Endocrinología y Nutrición, se publica desde abril de 1993. Creada como un órgano de difusión para la Sociedad Mexicana de Nutrición y Endocrinología, ha pasado por diferentes formatos, editoriales y procedimientos, que reflejan precisamente las dificultades de muchas revistas latinoamericanas para lograr el impacto que deberían tener las investigaciones regionales en los idiomas que nos caracterizan. Sin embargo, la calidad del trabajo editorial se ha mantenido desde el principio y la revista se está adaptando a los nuevos requerimientos científicos internacionales. Las mejoras aún están en proceso, lograr un índice de impacto además de la inclusión en otros índices son nuestros siguientes objetivos, entre otros más. El incremento en la calidad de la revista se debe a todos los colaboradores, la misma Sociedad Mexicana de Nutrición y Endocrinología, la editorial Permanyer, los autores que envían sus artículos, los revisores y los comentarios de nuestros lectores, por lo que agradecemos su continua y entusiasta participación.

\section{FINANCIAMIENTO}

La presente investigación no ha recibido ninguna beca específica de agencias de los sectores público, comercial, o sin ánimo de lucro.

\section{CONFLICTO DE INTERESES}

La Dra. Claudia Ramírez es coeditora de la revista.

\section{RESPONSABILIDADES ÉTICAS}

Protección de personas y animales. Los autores declaran que para esta investigación no se han realizado experimentos en seres humanos ni en animales. 
Confidencialidad de los datos. Los autores declaran que en este artículo no aparecen datos de pacientes.

Derecho a la privacidad y consentimiento informado. Los autores declaran que en este artículo no aparecen datos de pacientes.

\section{BIBLIOGRAFÍA}

1. Djulbegovic B, Guyatt GH. Progress in evidence-based medicine: a quarter century on. Lancet. 2017;390(10092):415-23.

2. Casadevall A, Fang FC. Specialized science. Infect Immun. 2014; 82(4):1355-60.

3. Bornmann L, Mutz R. Growth rates of modern science: A bibliometric analysis based on the number of publications and cited references. J Assic fir Information Sci Tech. 2015;66(11).

4. Sotudeh $\mathrm{H}$, Estakhr Z. Sustainability of open access citation advantage: the case of Elsevier's author-pays hybrid open access journals. Scientometrics. 2018;115:563.

5. Altman DG, Moher D. Declaration of transparency for each research article. BMJ. 2013;347:f4796.

6. Solomons NM, Spross JA. Evidence-based practice barriers and facilitators from a continuous quality improvement perspective: an integrative review. J Nurs Manag. 2011;19(1):109-20.
7. Baumann N. How to use the medical subject headings (MeSH). Int J Clin Pract. 2016;70(2):171-4.

8. Wiechert K, Wang JC, Chapman JR. Predator journals: An urgent and global problem. Global Spine J. 2019;9(3):253.

9. Relevo R, Balshem H. Finding evidence for comparing medical interventions. En: Agency for Healthcare Research and Quality, editores. Methods guide for effectiveness and comparative effectiveness reviews [Internet] Rockville (MD): Agency for Healthcare Research and Quality (US); 2008 [fecha de publicación: 5 de enero de 2011]. Disponible en: https://www. ncbi.nlm.nih.gov/books/NBK53479/

10. Filkins BL, Kim JY, Roberts B, Armstrong W, Miller MA, Hultner ML, et al. Privacy and security in the era of digital health: what should translational researchers know and do about it? Am J Transl Res. 2016;8(3):1560-80.

11. Lohr S. For impatient web users, an eye blink is just too long to wait. The New York Times. 29 de febrero de 2012. Disponible en: https://www. nytimes.com/2012/03/01/technology/impatient-web-users-flee-slowloading-sites.html

12. Lewandovski D. Google Scholar as a tool for discovering journal articles in library and information science. Online Info Rev. 2010;34(2):250-62.

13. Peroni S, Osborne F, Di lorio A, Nuzzolese AG, Poggi F, Vitali F, et al. Research articles in simplified HTML: a web-first format for HTML-based scholarly articles. PeerJ Computer Science, 2017; 3:e132.

14. Gorraiz J, Melero-Fuentes D, Gumpenberger C, Valderrama-Zurian JC. Availability of digital object identifiers (DOIs) in Web of Science and Scopus. J Infometrics. 2016;10(1):98-109.

15. Rosenblatt B. The Digital Object Identifier: Solving the dilemma of copyright protection online. J Electronic Pub. 1997;3(2):1-3.

16. Haddaway NR, Collins AM, Coughlin D, Kirk S. A rapid method to increase transparency and efficiency in web-based searches. Environ Evid. 2017;6(1): doi:10.1186/s13750-016-0079-2

17. Nell B, Lewis J. The World Wide Web. En: Nell B, Lewis J. Computer Science Illuminated, 2. a ed. Sudbury, MA, EE.UU.: Editorial Jones and Bartlett Publishers; 2002. pp. 505-522.

18. Tzitzikas Y, Marketakis Y. The File Poem.html: On Reading Characters. En Cinderella's Stick. Springer, Cham; 2018. pp. 39-47. 